\title{
Anthocyanin accumulation and differential gene expression in wild-type and mutant Syzygium malaccense fruits during their growth and ripening
}

\author{
P. KOTEPONG ${ }^{1 \dagger}$, R.E. PAULL ${ }^{2}$, and S. KETSA ${ }^{1,3 *}$ \\ Department of Horticulture, Faculty of Agriculture, Kasetsart University, Bangkok 10900, Thailand ${ }^{1}$ \\ Department of Tropical Plant and Soil Sciences, University of Hawaii, Manoa HI 96822, USA ${ }^{2}$ \\ Academy of Science, The Royal Society, Dusit, Bangkok 10300, Thailand ${ }^{3}$
}

\begin{abstract}
Ripe wild-type Malay apple (Syzygium malaccense) cv. Saraek fruit epidermis is red, whereas a mutant has a white skin. Wild-type and mutant fruit were used as a fruit model to study the regulation and gene expression patterns of anthocyanin biosynthesis and a myeloblastosis (MYB) transcription factor during growth and development. Fruit size, color, anthocyanin composition, and the expression of genes involved in anthocyanin biosyntheses were determined during fruit growth and ripening. Wild-type ripe fruit had a greater diameter and fruit mass than the mutant. The total anthocyanin content was approximately nine-fold higher in wild-type red fruit than in mutant white fruit. The major anthocyanin in the fruit skin of the wild-type was cyanidin-3-O-glucoside with minor amounts of pelargonidin-3-O-glucoside, peonidin-3- $O$-glucoside, and cyanidin-3,5-O-diglucoside. No anthocyanin was found in mutant fruit. The accumulation of cyanidin-3-O-glucoside during fruit growth and ripening was correlated with red color development and activities of phenylalanine ammonia lyase (PAL) and UDP-glucose:flavonoid-3-O-glucosyltransferase (UFGT). We cloned fragments and characterized seven genes involved in anthocyanin biosynthesis pathway namely phenylalanine ammonia lyase (SmPAL), chalcone synthase $(\mathrm{SmCHS})$, chalcone isomerase (SmCHI), flavanone-3-hydroxylase (SmF3H), dihydroflavonol 4-reductase (SmDFR), leucoanthocyanidin dioxygenase (SmLDOX), and UDP glucose-flavonoid-3-O-glucosyl transferase (SmUFGT), as well as a MYB transcription factor $(\operatorname{SmMYB})$. The expressions of all the genes were determined by semi-quantitative reverse transcription (RT)-PCR and quantitative real-time RT-PCR. The skin of wild-type fruit contained transcripts of all above mentioned genes, whereas the mutant fruit showed no SmUFGT and SmMYB expressions, which correlated with the absence of anthocyanin accumulation during fruit growth and ripening. These results suggest that lack of anthocyanin biosynthesis in mutant fruit may be via the regulation of $U F G T$ and $M Y B$ transcription factor expressions.
\end{abstract}

Additional key words: chalcone synthase, cyanidin-3-O-glucoside, Malay apple, MYB transcription factor, phenylalanine ammonia lyase, UDP glucose-flavonoid-3-O-glucosyl transferase.

\section{Introduction}

The genus Syzygium from the family Myrtaceae contains several species producing edible fruits. Their fruits are named 'apples' and indeed the crispiness of the fruit flesh is reminiscent of apples. The fruits of some commercially important species of $S$. are rather similar in form, but differ in taste. Depending on the cultivar, S. samarangense produces a green or pink skinned fruits, both called Java apple or Java rose apple whereas $S$. malaccense produces fruits called Malay apple, which has a dark red/purple skin. Synonyms used for Malay apple are Malacca apple or Malay rose apple. The Malay apple has an edible aril, which is white, juicy, sweet, and slightly-acid with a pleasant flavor, located inside a dark red to purple pericarp that is rich in bioactive secondary metabolites including

Submitted 17 February 2018, last revision 3 April 2019, accepted 16 April 2019.

Abbreviations: CHI - chalcone isomerase; CHS - chalcone synthase; DFR dihydroflavonol-4-reductase; F3GT - flavonol-3O-glucosyltransferase; F3H - flavanone-3-hydroxylase; LDOX - leucoanthocyanidin dioxygenase; MS - mass spectrometry; MYB - myeloblastosis; PAL - phenylalanine ammonia lyase; RT - reverse transcription; UFGT - UDP-glucose:flavonoid-3- $O$ glucosyltransferase.

Acknowledgements: The research was financially supported by the Thailand Research Fund (TRF) and the Southeast Asian Regional Center for Graduate Study and Research in Agriculture (SEARCA).

$\uparrow$ Present address: Postharvest and Processing Research and Development Division, Department of Agriculture, Ministry of Agriculture and Cooperatives, Bangkok 10900, Thailand

* Corresponding author; fax: (+66) 25791951 112, e-mail: agrsck@ku.ac.th 
anthocyanins, oligomeric proanthocyanins, and xanthones (Fu et al. 2007, Ji et al. 2007).

There are at least two cultivars of Malay apple grown in Thailand, called locally Mamieo and Saraek. Both cultivars are different in shape and color. 'Mamieo' is more ovoid and has a dark purple skin, whereas 'Saraek' has a stripe red skin. White mutants of both 'Mamieo' and 'Saraek' have been found in Thailand. Kotepong et al. (2011) reported that wild-type fruit of 'Mamieo' has a total anthocyanin content 30-times higher than white mutant fruit. Cyanidin-3-O-glucoside accounted for a large proportion of the total anthocyanin content.

Anthocyanins are water-soluble flavonoid pigments, which are found in higher plants and are responsible for red, blue, and purple color of many fruits, vegetables, flowers, and seeds. Anthocyanin biosynthesis involves a common branch of the flavonoid pathway and has been intensively investigated in many plants including Arabidopsis, petunia, maize, and grape. The regulation of anthocyanin biosynthesis is mainly at the level of transcription of the structural genes by transcription factors. The structural genes of the anthocyanin biosynthetic pathway have been isolated from many fruit and include phenylalanine ammonia lyase (PAL), chalcone synthase (CHS), chalcone isomerase (CHI), flavanone3-hydroxylase $(F 3 H)$, flavonoid-3'-hydroxylase $\left(F 3^{\prime} H\right)$, dihydroflavonol-4-reductase (DFR), leucoanthocyanidin reductase (LDOX), and UDP-glucose:flavonoid-3-Oglucosyltransferase (UFGT) (Winkel-Shirley 2002). In apple, analysis of transcript abundance of these genes during fruit development indicates that the expressions of most of the genes in the pathway $(C H S, F 3 H, D F R$, $L D O X$, and $U F G T$ ) are coordinated during anthocyanin accumulation in apple skin (Honda et al. 2002). The expression pattern in apple differs from that in grape (Vitis vinifera and $V$. labruscana), in which UFGT induction during anthocyanin accumulation is a key regulatory step in coloration (Kobayashi et al. 2001, Zhang et al. 2013). In mangosteen fruit, GmMYB10 appears to play a role in the specific regulation of anthocyanin biosynthesis both ontree and off-tree. The GmUFGT may be a key biosynthetic gene in mangosteen pigmentation (Palapol et al. 2009a). The transcription factors which control anthocyanin biosynthesis include myeloblasosis (MYB) transcription factors, basic helix-loop-helix transcription factors, and a repeat-containing protein WD40 (Broun 2005, Koes et al. 2005). Plant MYB transcription factors play important roles in many secondary metabolic pathways including anthocyanin and flavonol. Myeloblastosis transcription factors are classified by conserved imperfect repeats in the DNA-binding domain, termed R1, or R2R3. There are 126 R2R3 MYB genes in Arabidopsis (Stracke et al. 2001) and 108 in grape (Matus et al. 2008). In grape, mutation were found in two genes encoding MYB transcription factors that regulate flavonol-3-O-glucosyltransferase (F3GT) expression (Kobayashi et al. 2004, Walker et al. 2007).

The aim of this work was to compare content of anthocyanins, which are associated with color development, and their composition in fruit skin of wild-type and mutant Malay apple. We tried to show how anthocyanins accumulation at different fruit stages is related to enzyme activities and expressions of anthocyanin biosynthetic genes and the MYB transcription factor. We tested the hypothesis that lack of red color in the mutant is due to lack of a regulatory gene in anthocyanin biosynthetic pathway.

\section{Materials and methods}

Fruit collection: The white mutant tree of Malay apple (Syzygium malaccense (L.) Merr. \& L.M. Perry) 'Saraek' was found naturally by a grower who planted the wild type of Malay apple, which were propagated by layering. Malay apple with red (wild-type) and white (mutant) skin were obtained from the same orchard in the Nonthaburi province of central Thailand. Trees of both wild type and mutant of Malay apple were about 20 years old and were approximately $3 \mathrm{~m}$ high. Flowers were tagged at full bloom. Fruits were bagged at stage 2 (Fig. 1 Suppl.). Samples of 30 fruits were collected randomly from several trees throughout the growing season. Developmental stages were divided into 8 stages: stage $1-21 \mathrm{~d}$ after full bloom (DAFB); stage 2 - 28 DAFB; stage 3 - 35 DAFB; stage 4 - 42 DAFB; stage 5 - 49 DAFB; stage 6 - 52 DAFB; stage 7 - 55 DAFB, and stage 8 - 58 DAFB which is a fully ripe stage (Fig. 1 Suppl.). Stage 7 is the normal picking stage for export markets whilst fruit is picked at stage 8 if sold at local markets. After harvest, fruits were transported in an air-conditioned $\operatorname{car}\left(25^{\circ} \mathrm{C}\right)$ to the laboratory within $2 \mathrm{~h}$.

Fruit growth was determined at each fruit developmental stage. An individual fresh mass was measured using a digital balance ( $A N D$, Tokyo, Japan). Fruit width and fruit diameter were measured using a digital vernier caliper (Insize, GA, USA). Fruit skin color was measured using a CR-300 Chromameter (Minolta, Osaka, Japan) as $\mathrm{L}^{*}$, $\mathrm{a}^{*}$, and $\mathrm{b}^{*}$ values (the CIE color system, Selahle et al. 2015) and converted to a hue angle (a color wheel with red-purple at an angle of $0^{\circ}$, yellow at $90^{\circ}$, and bluishgreen at $180^{\circ}$ ). Both high $\mathrm{a}^{*}$ and high $\mathrm{b}^{*}$ values and low hue angles describe the intensity of red and yellow colors of the material whereas $\mathrm{L}^{*}$ values describe its lightness (Selahle et al. 2015). The color reading was taken twice at the top, middle, and bottom regions of each fruit and then averaged to estimate a value for each fruit. After fruit skin color measurement, the fruit skin was peeled off carefully with a knife. The samples of the fruit skin were frozen immediately in liquid nitrogen and stored at $-86 \mathrm{C}$ until used for further experiments.

Anthocyanin analysis: Total anthocyanins were extracted from fruit skin according to the method described by Piccaglia et al. (2002). One gram of each sample was homogenized with $20 \mathrm{~cm}^{3}$ of methanol $(99.9 \%)$ plus $\mathrm{HCl}$ (37\%) (99:1, v/v) using a Polytron PT 2100 (Kinematica, Luzern, Switzerland) with a $1.2 \mathrm{~mm}$ diameter head at a speed of 12 for $1 \mathrm{~min}$ and then shaken at $4{ }^{\circ} \mathrm{C}$ in darkness for $6 \mathrm{~h}$. The aqueous phase was removed and the pellet was re-extracted four times with $20 \mathrm{~cm}^{3}$ of methanol (99.9\%) plus $\mathrm{HCl}(37 \%)(99: 1, \mathrm{v} / \mathrm{v})$ within $24 \mathrm{~h}$ and then adjusted 
to a final volume of $100 \mathrm{~cm}^{3}$ with the same solution. The combined aqueous extracts were centrifuged at $8000 \mathrm{~g}$ and $4{ }^{\circ} \mathrm{C}$ for $10 \mathrm{~min}$ to remove the pellet. The absorbance of the supernatant was measured at $530 \mathrm{~nm}$ using a Genesys $10 U V$ spectrophotometer (Thermo Spectronic, Rochester, NY, USA). Total anthocyanin content was expressed as cyanidin equivalents (a molar absorbance of 34.3). All measurements were made in triplicate. The samples were kept in an amber bottle at $-86^{\circ} \mathrm{C}$ until individual anthocyanins were quantitified.

Individual anthocyanins were analyzed by HPLC and LC tandem mass spectrometry (MS/MS), using a modified method described by Stevenson et al. (2006). The samples were filtered through a syringe filter, a 0.45 $\mu \mathrm{m}$ nylon membrane (Chromtech, Apple Valley, MN, USA) before injection. Individual samples were analyzed using a Shimadzu analytical HPLC with a column oven, auto-sampler injector, vacuum solvent degas module, and $L C$-10AD VPdetector(Shimadzu, Kyoto, Japan). Separation was performed on two connected columns. The first was a guard column to filter out any contamination (using a $3 \mathrm{~mm}$ diameter security guard cartridge; Phenomenex, Torrance, CA, USA), the second was a Synergi $250 \times$ $4.6 \mathrm{~mm}$ column filled with Polar RP $4 \mu \mathrm{m}$ diameter particles, $8 \mathrm{~nm}$ pore size (Phenomenex). A gradient of two mobile phases was used: A: acetonitrile $(99.9 \%)+$ formic acid $(98 \%)(99: 1, \mathrm{v} / \mathrm{v})$ and $\mathrm{B}$ : acetonitrile (99.9\%) + water + formic acid $(98 \%)(5: 92: 3, \mathrm{v} / \mathrm{v} / \mathrm{v})$. A flow rate was $1 \mathrm{~cm}^{3} \mathrm{~min}^{-1}$ at a column temperature of $45^{\circ} \mathrm{C}$. The gradient consisting of the mobile phase A was $0 \%$ at 0 min and ramped linearly to $20 \%$ at $20 \mathrm{~min}$, to $30 \%$ at $26 \mathrm{~min}$, to $50 \%$ at $28.5 \mathrm{~min}$, to $95 \%$ between 32 and $35 \mathrm{~min}$, and back to $0 \%$ between 36 and $42 \mathrm{~min}$. The individual anthocyanins were identified at $520 \mathrm{~nm}$ using the retention times and by comparison with standard solutions of known concentrations.

The LC-MS/MS analysis of samples at stage 8 (Fig. 1 Suppl.) was carried out using a mass spectrometer (Bruker, Bellerica, MA, USA) equipped with an electrospray ionization source. Separation was performed on a $150 \times$ $4.6 \mathrm{~mm}$ column, 2 XHypersil Gold, $3 \mu \mathrm{m}$ particle diameter. The anthocyanin fractions were analyzed in the positive ion mode using both a molecular ion mass $(\mathrm{M}+)$, and a tandem mass spectrogram (MS/MS) of the fractions at $530 \mathrm{~nm}$, and comparison of retention times and fragmentations with an authentic commercial standard of cyanidin-3-O-glucoside (Polyphenols, Sandnes, Norway).

Activities of PAL and UFGT: Enzymes were extracted from fruit skin according to Lister et al. (1996a) with a slight modification. The frozen fruit skin ( $3 \mathrm{~g})$ at each stage was homogenized in $15 \mathrm{~cm}^{3}$ of $50 \mathrm{mM} \mathrm{Na} \mathrm{HPO}_{4} / \mathrm{KH}_{2} \mathrm{PO}_{4}$ buffer ( $\mathrm{pH}$ 7.0) containing $50 \mathrm{mM}$ sodium ascorbate, $18 \mathrm{mM}$ 2-mercaptoethanol, $2 \mathrm{~g}$ of polyvinylpyrrolidone, and $0.1 \%(\mathrm{v} / \mathrm{v})$ Triton $X-100$. The sample was homogenized using a Polytron PT 2100 (Kinematica, Luzern, Switzerland) with a $1.2 \mathrm{~mm}$ diameter head at a speed of 12 for $1 \mathrm{~min}$. The sample was incubated on ice for $10 \mathrm{~min}$, then centrifuged at $20000 \mathrm{~g}$ for $20 \mathrm{~min}$ to remove particulate matter. Ammonium sulphate was added to the supernatant at $35 \%$ saturation followed by centrifugation at $20000 \mathrm{~g}$ for $20 \mathrm{~min}$ to remove polyvinylpyrrolidone, and more ammonium sulfate was added to reach a final saturation of $80 \%$. This fraction was centrifuged at $20000 \mathrm{~g}$ for $20 \mathrm{~min}$. Afterwards, the pellet was re-suspended in $3 \mathrm{~cm}^{3}$ of the homogenization buffer without polyvinylpyrrolidone and Triton $X-100$ and dialyzed with a dialysis buffer (the homogenization buffer without polyvinylpyrrolidone and Triton $X-100$ ) overnight to give a partially purified extract, which was used for PAL and UFGT enzyme activities. All enzyme extractions steps were carried out at $4{ }^{\circ} \mathrm{C}$. The enzyme extract was frozen in liquid nitrogen and stored at $-86^{\circ} \mathrm{C}$ until enzyme activity analyses.

Activity of PAL was measured using the method of Lister et al. (1996a) with a modification. A reaction mixture comprised $0.2 \mathrm{~cm}^{3}$ of the enzyme extract and $0.8 \mathrm{~cm}^{3}$ of $60 \mathrm{mM}$ borate buffer $(\mathrm{pH} 8.8)$. The reaction was initiated by addition of $0.2 \mathrm{~cm}^{3}$ of L-phenylalanine solution $\left(10 \mathrm{mg} \mathrm{dm}^{-3}\right)$ and incubated at $30^{\circ} \mathrm{C}$ in a water bath for $1 \mathrm{~h}$. The reaction was stopped by adding $125 \mathrm{~mm}^{3}$ of $40 \%(\mathrm{~m} / \mathrm{v})$ trifluoroacetic acid and then centrifuged at $12000 \mathrm{~g}$ and $4{ }^{\circ} \mathrm{C}$ for $5 \mathrm{~min}$ to pellet the denatured protein. Activity of PAL was assesed by measuring absorbance at $290 \mathrm{~nm}$ using a Genesys 10UV spectrophotometer (Thermo Spectronic, Rochester, NY, USA). Triplicate assays were performed for each enzyme extract. One unit of PAL was defined as the yield of $1 \mu \mathrm{mol}$ of trans-cinnamic acid per $\mathrm{mg}$ of protein.

Activity of UFGT was measured using the method of Lister et al. (1996b) with a modification. A reaction mixture comprised $0.2 \mathrm{~cm}^{3}$ of the enzyme extract, $0.2 \mathrm{~cm}^{3}$ of $50 \mathrm{mM}$ bicine buffer $(\mathrm{pH} 8.5), 30 \mathrm{~mm}^{3}$ of $1 \mathrm{mM}$ quercetin, and $20 \mathrm{~mm}^{3}$ of $2.5 \mathrm{mM}$ UDP-glucose. The reaction mixture was incubated in a water bath at $30{ }^{\circ} \mathrm{C}$ for $30 \mathrm{~min}$. The reaction was halted by addition of $0.15 \mathrm{~cm}^{3}$ of $20 \%(\mathrm{~m} / \mathrm{v})$ trichloroacetic acid in methanol $(100 \%)$ and then centrifuged at $12000 \mathrm{~g}$ and $4{ }^{\circ} \mathrm{C}$ for $5 \mathrm{~min}$. The supernatant was stored at $-86^{\circ} \mathrm{C}$ until quantification by HPLC. The samples were filtered through a syringe filter, a $0.45 \mu \mathrm{m}$ nylon membrane (Chromtech, Apple Valley, MN, USA) before injections. Analysis of quercetin-3-O-glucose

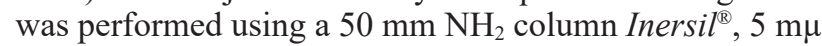
particle diameter, with a $4.0 \times 10 \mathrm{~mm}$ HPLC guard column, Inersil $^{\circledR} O D S-3,5 \mu \mathrm{m}$ particle diameter. A flow rate was $1 \mathrm{~cm}^{3} \mathrm{~min}^{-1}$ at a column temperature of $30^{\circ} \mathrm{C}$. An injection volume was $20 \mathrm{~mm}^{3}$. Mobile phase were: A: acidified water (0.66 M formic acid) and B: acidified methanol (0.66 M formic acid in $100 \%$ methanol) in a linear gradient, where A:B ratio changed from 50:50 to $15: 85$ during the first $5 \mathrm{~min}$ followed by an isocratic mixture for $1 \mathrm{~min}$ and back to the initial conditions. The product was detected at $350 \mathrm{~nm}$ and compared with a standard (quercetin3-O-glucoside). One unit of UFGT was defined as the production of $1 \mathrm{~mol}$ quercetin-3-O-glucoside per second. Protein content in the enzyme extract was measured using the method described by Bradford (1976) with bovine serum albumin as a standard. 
Extraction of RNA and cDNA synthesis: Approximately $5 \mathrm{~g}$ of frozen samples were ground in a Retsch MM 301 mixing mill (Retsch, Newtown, PA, USA). Total RNA was isolated from $2 \mathrm{~g}$ of fruit skin tissue as described by Lopez-Gomez and Gomez-Lim (1992). The total RNA was treated with DNase I (RNase free, Fermentas, Burlington, Canada). The first strand cDNA was synthesized from $4 \mu \mathrm{g}$ of total RNA following the protocol of an Omniscript RT kit (Qiagen, Hilden, Germany) as a template for semiquantitative RT-PCR and quantitative real-time RT-PCR.

Isolation of anthocyanin biosynthesis genes and a MYB transcription factor: Athocyanin biosynthesis genes and a MYB transcription factor were isolated from stage 8 of wild-type fruit skin using forward and reverse degenerate primers (Table. 1 Suppl.) based on conserved regions of similar genes in the NCBI database. The amplification reactions of anthocyanin biosynthesis genes were as follows: $94^{\circ} \mathrm{C}$ for $30 \mathrm{~s}$ and $5 \mathrm{~min}$ and then $54{ }^{\circ} \mathrm{C}$ for $30 \mathrm{~s}(\mathrm{SmPAL}, \mathrm{SmCHI})$ or $56^{\circ} \mathrm{C}$ for $30 \mathrm{~s}$ (SmDFR, SmCHS, $S m F 3 H, S m L D O X$, and $S m U F G T$ ) with a final period at $72{ }^{\circ} \mathrm{C}$ for $30 \mathrm{~s}$ and $10 \mathrm{~min}$. For the isolation of a MYB transcription factor, degenerate primers and amplification reactions were carried out as described by Takos et al. (2006).

The amplified PCR fragments from each gene were purified using a gel extraction kit (QIAquick ${ }^{\mathbb{R}}$ gel extraction, Qiagen, Hilden, Germany) and cloned into a pGEM-T vector ( $p G E M^{\circledR}-T$ Easy, Promega, Fitchburg, WI, USA). Transformation of the vector with the inserted DNA was conducted using Escherichia coli strain DH5 $\alpha$ cells. Plasmid DNA containing the vector and inserted DNA was extracted using a miniprep kit (QIAprep ${ }^{\circledR}$ Spin Miniprep kit, Qiagen). The sequence analysis of the clone was conducted by automatic sequencing using an $A B I$ PRISM ${ }^{\circledR} 377$ DNA sequencer (Applied Biosystems, Foster City, CA, USA). The sequences of all amplified fragments were compared with the genes in the Genbank database using the BLAST program from NCBI. A new pair of specific primers for the gene were encoded for all genes (Table 1 Suppl.) and used for semi-quantitative RT-PCR and real-time quantitative PCR. Specific primers were tested for specificity using plasmid amplification and the product analyzed on a $1.2 \%(\mathrm{~m} / \mathrm{v})$ agarose gel stained with ethidium bromide. All primers were designed with Primer3 (v. 0.4.0; http://frodo.wi.mit.edu/primer3). The size of quantitative PCR products ranged from 121 to 181 bp (Table 2 Suppl.).

Gene expressions: Gene expressions were analyzed using real time quantitative PCR. Fifty-fold diluted cDNA was used. Quantitattive PCR was performed using an $A B I$ Prism $^{\circledR} 7000$ real-time PCR machine (Applied Biosystems, Foster City, CA, USA) using SYBR green (QuantiTectTM SYBR ${ }^{\circledR}$ Green PCR kit, Qiagen, Hilden, Germany) following the manufacturer's instructions. All reactions were performed in triplicate using $3 \mathrm{~mm}^{3}$ of diluted template $(50 \times), 1 \mathrm{~mm}^{3}$ of each forward and reverse primers $(2.5 \mu \mathrm{M})$, and $12.5 \mathrm{~mm}^{3}$ of $2 \times$ Master mix to a final volume of $25 \mathrm{~mm}^{3}$. Reactions were initiated at $95{ }^{\circ} \mathrm{C}$ for
$15 \mathrm{~min}$ followed by 40 cycles of $95^{\circ} \mathrm{C}$ for $5 \mathrm{~s}, 60^{\circ} \mathrm{C}$ for $5 \mathrm{~s}$, $72^{\circ} \mathrm{C}$ for $30 \mathrm{~s}$ and completed by a melting curve analysis. A negative water control and a melting curve were included in every run. A melting peak, a dissociation curve, and sequencing were analyzed to confirm the expected product rather than the primer dimmer. The data were normalized to actin transcription (SmACT) to minimize variation in cDNA template levels.

Statistical analysis: Experimental data were analyzed statistically by $A N O V A$, and significance of the differences between means was estimated by the Duncan's new multiple range test (DMRT) at $\alpha=0.05$. Experimental data are averages of three replications \pm standard errors. All experiments were repeated at least twice.

\section{Results}

All Malay apple fruits were separated into eight stages of development. Wild-type fruits at stage 4 of development had a red striped skin at the proximal part of the fruit. The streaked red color subsequently covered the remainder of the fruit. The color became darker over time, and fruits were dark red at stage 8 (Fig. 1 Suppl.). Fruit of the mutant had a light green color from stage 1 to stage 4 . The green changed to white or creamy with a very light pink during stages 5 to 8 (Fig. 1 Suppl.). Both wild-type and mutant fruits had characteristic shapes. Mutant fruit was more ovoid than the wild-type fruit. The fruits of the wild-type tended to have a greater diameter (Fig. 1A-D), height (Fig. $1 B-E$ ), and mass (Fig. $1 C-F$ ) than those of the mutant.

The $L^{*}$ values of wild-type fruits remained stable from stage 1 to stage 3 and then decreased sharply during maturation and ripening (stages 6 to 8), whereas L* values of mutant fruits slightly increased from stage 1 to stage 8 (Fig. 2A-E). The a* values of both wild-type fruits and mutant fruits did not change during stages 1 to 3 and then increased rapidly in wild-type fruits and increased steadily in mutant fruits from stage 4 to stage 8 (Fig. $2 B-F$ ). Mutant fruits had higher $b^{*}$ values than wild-type fruits from stage 1 to stage 7 , but no significant difference at stage 8 (Fig. 2C-G). Mutant fruits also had higher hue values than wild-type fruits from stage 5 to stage 8 (Fig. $2 D-H$ ).

A considerably higher total anthocyanin content was observed in wild-type fruits than in mutant fruits at all stages of growth except stage 1 (Fig. $3 A-B$ ). When fruits were ready to harvest (stage 7 ), the total anthocyanin content of wild-type fruits was 5-times higher than that of mutant fruits, whereas at the ripe stage (stage 8), wildtype fruits had a 9-times higher total anthocyanin content (Fig. 3A-B).

Four anthocyanins were detected in the fruit skin with similar retention times to the standards (Fig. $2 A$ Suppl., Table 3 Suppl.). The major anthocyanin in the skin of ripe fruits was cyanidin-3-O-glucoside with the minor content of pelargonidin-3-O-glucoside, peonidin-3-O-glucoside, and cyanidin-3,5-O-diglucoside (Fig. $2 A$ Suppl., Fig. 3 Suppl. and Table 3 Suppl.). No anthocyanins were found in the epidermis of mutant fruits (Fig. $2 B$ Suppl.). The 
major anthocyanin cyanidin-3-O-glucoside content in the epidermis of wild-type fruits increased rapidly during growth and ripening (Fig. 4).

Activities of PAL in the fruit skin of both wild-type fruits and mutant fruits were comparable and increased continuously from stage 1 to 8 (Fig. $5 A-C$ ). Activity of UFGT in the skin of wild-type fruits increased steadily from stage 1 to stage 8 whereas UFGT activity in the skin of mutant fruits was below the detection limit at all stages of fruit growth (Fig. $5 B-D$ ).

From the skin of wild-type fruits at stage 8 , we isolated seven partial coding sequences of genes involved in the anthocyanin biosynthetic pathway: SmPAL (accession No. GU233756; 381 bp; 126 amino acids), SmCHS (accession No. GU233757; 550 bp; 182 amino acids), SmCHI (accession No. GU233758; 484 bp; 161 amino acids), SmF3H (accession No. GU233759; 794 bp; 264 amino acids), SmDFR (accession No. 233760; 574 bp; 191 amino acids), SmLDOX (accession No. GU233761; 580 bp; 192 amino acids), and SmUFGT (accession No. GU233762; 947 bp; 351 amino acids) and a MYB transcription factor: SmMYB (accession No. HM773023; 249 bp; 83 amino acids). The possibility that differences in anthocyanin biosynthesis during growth and ripening in Malay apple were related to gene expression patterns was investigated. Seven anthocyanin-related genes were isolated by PCR from $S$. malaccense cDNA templates. All genes showed a high homology to other plant anthocyanin biosynthetic genes (Table 4 Suppl.). The Malay apple SmPAL, SmCHS, and SmCHI translated protein sequences showed the highest homology with Pyrus pyrifolia: a $72-97 \%$ amino acid identity. The Malay apple SmF3H, SmDFR, SmLDOX, and SmUFGT showed a 55 - $91 \%$ identity to Pyrus cerasifera (Table 4 Suppl.). The SmUFGT involved in the last step of glucose-based anthocyanin biosynthetic pathway showed a somewhat lower identity $(55-63 \%)$ at the amino acid level in comparison with other species (Table 4 Suppl.). Myeloblastosis transcription factors showed a $85-88 \%$ identity with red fruits of cherry plum, grape, litchi, mangosteen, and strawberry (Table 4 Suppl.).

Transcriptions of all seven genes were detectable in the skin of both wild-type fruits and mutant fruits (Fig. 6) with the exception of SmUFGT in the mutant, which was below the detection limit (Fig. $6 N$ ). In the wild-type, the transcript abundance of SmPAL increased slightly during early fruit growth, and a high transcript abundance was found during ripening (Fig. 6A). In mutant fruits, the transcript abundance of $S m P A L$ remained stable during fruit growth and ripening (Fig. $6 H$ ). The transcript abundances of SmCHS, SmCHI, SmF3H, SmDFR, and $S m L D O X$ were similar in wild-type and mutant fruits (Fig. $6 B-F, I-M$ ). In wild-type fruits, SmUFGT transcription gradually increased from stage 3 to stage 7 and then increased rapidly at stage 8 with a more than an 80 -fold increase (Fig. 6G). In the wild-type, the transcription of

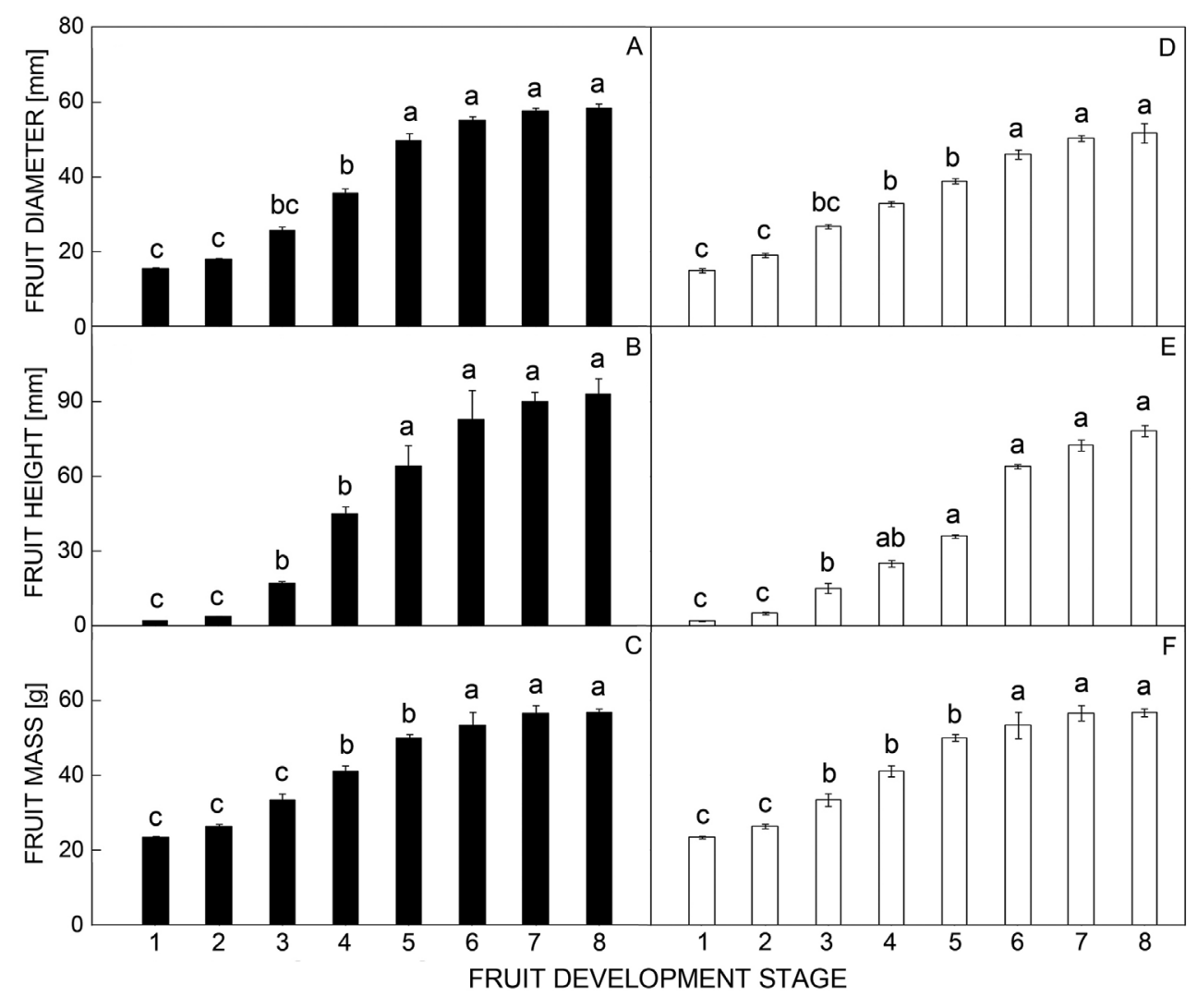

Fig. 1. Changes in diameter $(A, D)$, height $(B, E)$, and mass $(C, F)$ of wild-type $(\mathbf{\square})$ and mutant $(\square)$ Malay apple fruits during their growth and ripening. Means \pm SEs, $n=3$. Different letters indicate significant differences according to the Duncan's new multiple range test $(P<0.05)$. 
the $S m M Y B$ showed a more than 28-fold gradual increase from stage 3 to stage 7 and then a decrease at stage 8
(Fig. 7A). In mutant fruits, the transcript abundance of $S m M Y B$ remained very low (Fig. $7 B)$.

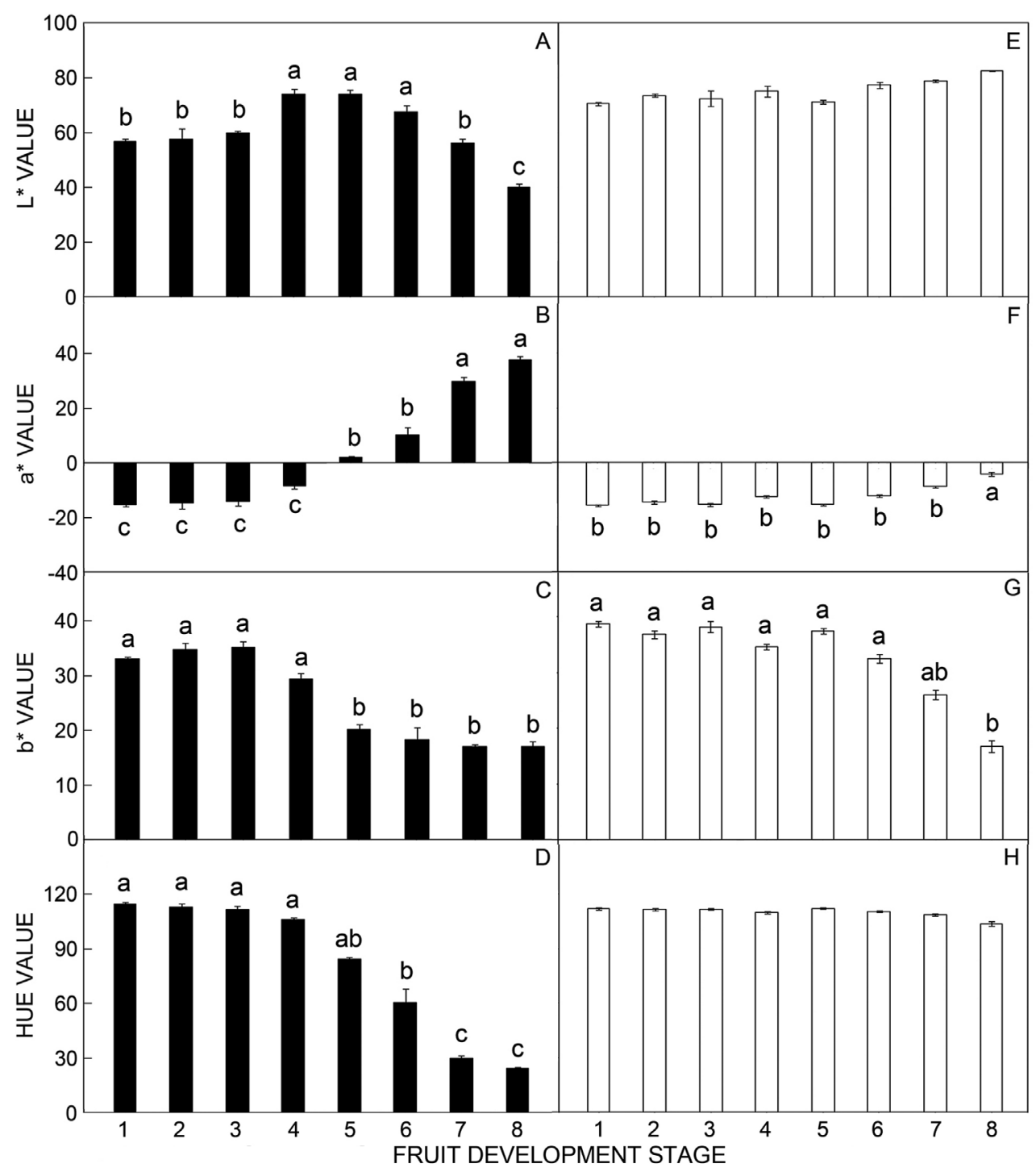

Fig. 2. Changes in $\mathrm{L}^{*}(A, E), \mathrm{a}^{*}(B, F), \mathrm{b}^{*}(C, G)$, and hue $(D, H)$ values of wild-type $(\square)$ and mutant $(\square)$ Malay apple fruits during their growth and ripening. Means \pm SEs, $n=3$. Different letters indicate significant $(P<0.05)$ differences according to the Duncan's new multiple range test.

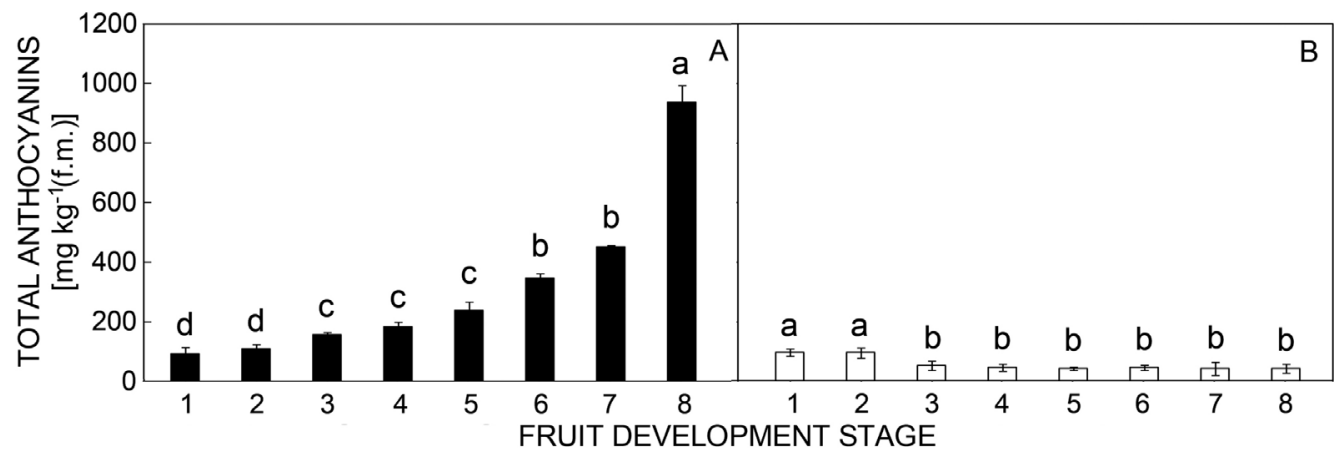

Fig. 3. Changes in total anthocyanin content in the skin $(A, B)$ of wild-type (匹) and mutant ( $\square$ ) Malay apple fruits during their growth and ripening. Means \pm SEs, $n=3$. Different letters indicate significant differences according to the Duncan's new multiple range test $(P<0.05)$. 


\section{Discussion}

During fruit growth, we observed a large increase in skin anthocyanin content in wild-type fruits from the early stage to the ripe stage (stages $1-8$ ). Ripe wild-type fruits had a much higher skin anthocyanin content than ripe mutant fruits. Higher skin anthocyanin content of ripe wild-type fruits was related to the deepness of the red color. This was reflected in the measured lower $b^{*}, L^{*}$ and hue values and higher $a^{*}$ values in the wild-type compared to the mutant. A similar increase of anthocyanin content during ripening has also been reported in many other fruits such as avocado (Ashton et al. 2006 ), lychee (Cronje 2008), apple (Awad and De Jager 2002), blueberry (Kalt et al. 2003), muscadine grape (Lee and Talcott 2004), grape (Segade et al. 2008), mangosteen (Palapol et al. 2009b), and mango (Sivankalyani et al. 2016). Ripe wild-type fruits of Malay apple 'Mamieo' with a dark purple skin contain total anthocyanin content about 3 times higher than 'Saraek' (Kotepong et al. 2011). This suggests that anthocyanin accumulation in wild-type fruits correlates well with red

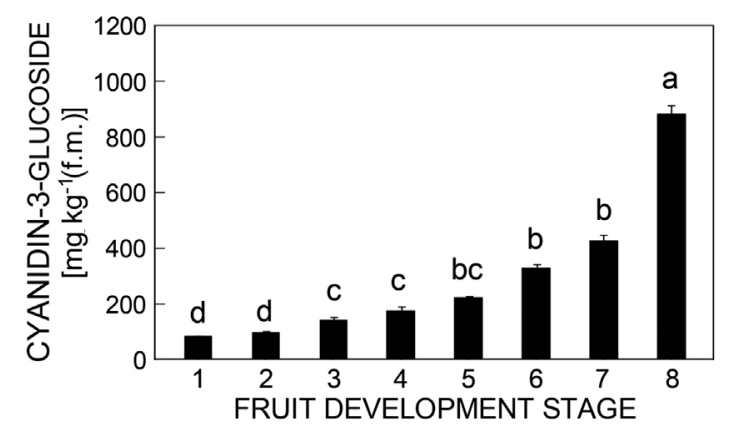

Fig. 4. Changes in cyanidin-3-O-glucoside content of the skin of wild-type Malay apple fruits during their growth and ripening. Means \pm SEs, $n=3$. Different letters indicate significant differences according to the Duncan's new multiple range test $(P<0.05)$ color development. Although the fruits of the white mutant at stage 1 contained the amount of anthocyanins similar to the wild-type, a very low anthocyanin content was found at further stages of growth and ripening.

The major anthocyanin found in the skin of wild-type fruits was cyanidin-3-O-glucoside confirming that already reported (Kotepong et al. 2011). Its increase accounted for most of the increase in the total anthocyanin content during the late stages of fruit growth and ripening. Red color development in the wild-type fruit skin was closely correlated with the strongly increasing content of cyanidin-3-O-glucoside. Cox et al. (2004) reported a similar increase in cyanidin-3-O-glucoside content during avocado ripening, which correlates with red coloration of the skin. The major anthocyanins in avocado also include cyanidin-3-O-glucoside (Ashton et al. 2006). In apple, the content of cyanidin-3-galactoside increases during maturation and ripening coinciding with an increase in red blush (Whale and Singh 2007). Small amounts of the other anthocyanins including pelargonidin-3-O-glucoside, peonidin-3-O-glucoside, and cyanidin-3,5-O-diglucoside were also detected in the fruit skin of wild-type Malay apple. No anthocyanin was found in the mutant fruit skin. In the absence of other pigments, the dramatic increase in content of cyanidin-3-O-glucoside alone explains the final red appearance of the wild-type fruits. The PAL and UFGT are important enzymes in anthocyanin biosynthesis (Xie et al. 2011). The activities of PAL and UFGT in the wildtype fruit skin continuously increased during fruit growth and ripening. Both PAL and UFGT activities showed positive correlations with the total anthocyanin content of red wild-type fruit. Activity of PAL was also found in the white mutant skin. This indicates that PAL may not be a key enzyme regulating anthocyanin synthesis in white mutant fruit of Malay apple, which is similar to that reported for apple (Liu et al. 2013). The increase in PAL activity in red skin fruit during fruit growth and ripening was involved in an increase of anthocyanin biosynthesis whereas PAL

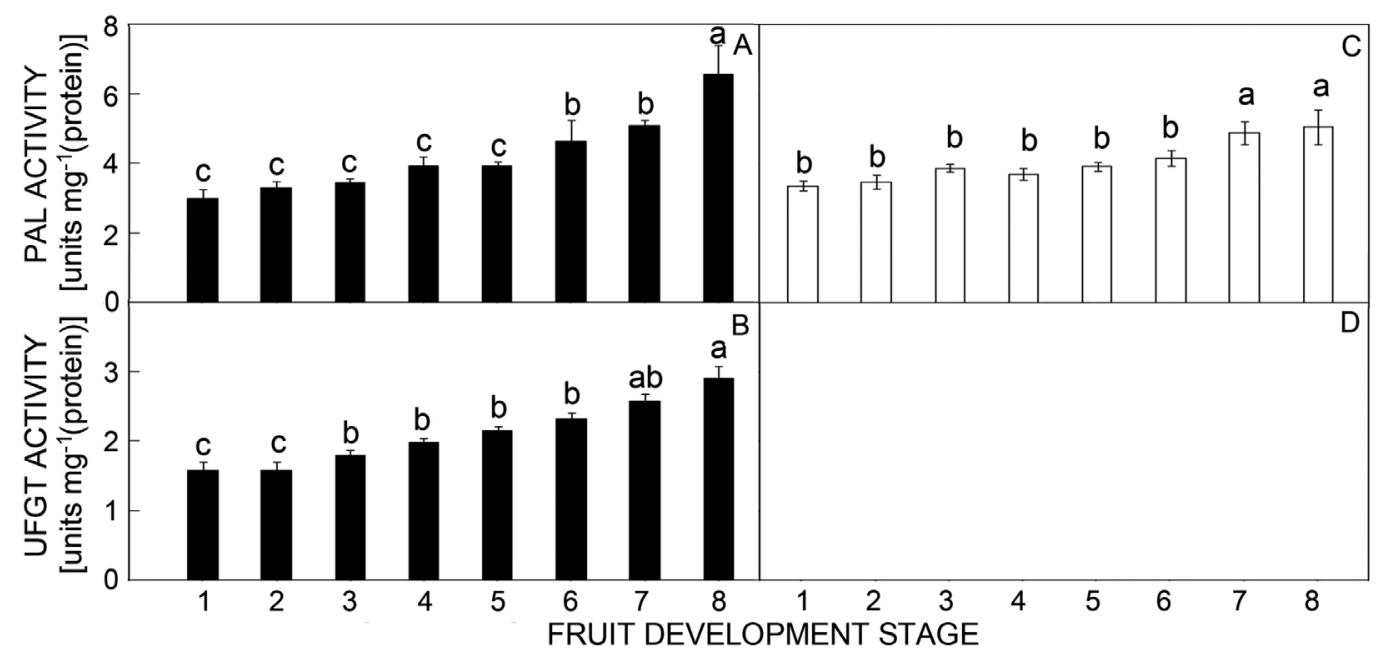

Fig. 5 Phenylalanine ammonia lyase (PAL) $(A, C)$ and UDP-glucose:flavonoid-3-O-glucosyltransferase (UFGT) $(B, D)$ activities in the skin of wild-type ( $\bullet$ and mutant( $\square$ ) of Malay apple fruits during their growth and ripening. Means \pm SEs, $n=3$. Different letters indicate significant differences according to the Duncan's new multiple range test $(P<0.05)$. 
activity showed in white skin fruit may be involved in biosynthesis of other phenolic compounds. The PAL is the first enzyme in the phenylpropanoid pathway leading to a wide range of compounds, such as phenols, anthocyanins, flavonoids, and lignins, which are abundant in apple skin (Vogt 2010). Nevertheless, anthocyanins are probably the major phenolic compounds being synthesized in red skin of many fruits during ripening, and the total anthocyanin

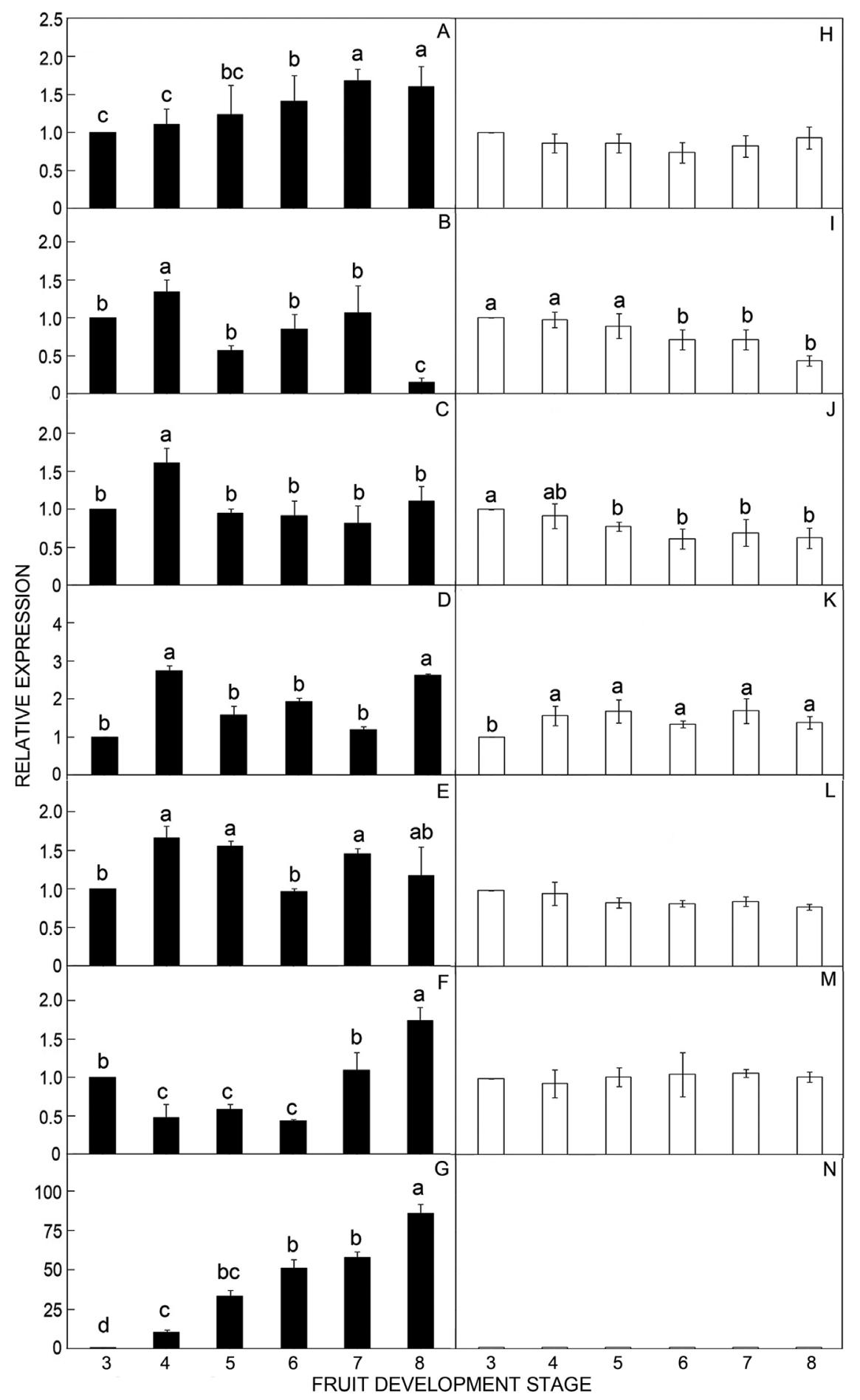

Fig. 6. Relative expressions of anthocyanin biosynthesis genes in Malay apple wild-type (®) and mutant ( $\square$ ) fruits during their growth and ripening. Real-time PCR was used to analyze phenylalanine ammonia lyase $(A, H)$, chalcone synthase $(B, I)$, chalcone isomerase $(C, J)$, flavanone-3-hydroxylase $(D, K)$, dihydroflavonol-4-reductase $(E, L)$, leucoanthocyanidin dioxygenase $(F, M)$, and $U D P$ glucose:flavonoid-3-O-glucosyltransferase $(G, N)$ expression patterns. The column heights indicate relative mRNA abundances relative to stage 3, which was set to 1.0. All real time-PCR reactions were normalized using the actin gene. Means \pm SEs, $n=3$. Different letters indicate significant differences according to the Duncan's new multiple range test $(P<0.05)$. 
content correlated well with PAL activity. According to Ju et al. (1995b), PAL catalyzes a reaction producing precursors of anthocyanin synthesis under conditions of sufficient resources, but changes in anthocyanin accumulation can occur independently of changes in PAL activity. In ripe apples, anthocyanin accumulation decreased even though PAL activity was relatively high. Therefore, the maximum PAL activity is not the regulating factor for anthocyanin accumulation in ripe apple (Wang et al. 2000).

Activity of UFGT was also positively correlated with anthocyanin accumulation during fruit growth and ripening in wild-type Malay apple fruit. Similarly, changes in UFGT activity parallel those in PAL activity in ripening grape (Mori et al. 2004). These data suggested a parallel upregulation of the activities of several enzymes involved in anthocyanin biosynthesis. Our data furthermore suggest that the absence of UFGT activity in the mutant fruit skin did not block PAL activity. Although a correlation is often found between PAL activity and anthocyanin synthesis in fruit skin, PAL activity is not the regulating factor for anthocyanin accumulation in the fruit (Wang et al. 2000). Our data also suggest independence in the regulation of PAL and UFGT activities.

Ju et al. (1995a) reported that the importance of UDPgalactose:flavonoid-3-O-glucosyltransferase in regulating anthocyanin biosynthesis in apples probably depends on the availability of its precursor, namely cyanidin. In strawberry fruit, changes in UFGT activity has been shown to parallel with PAL activity (Xu et al. 2014). This suggests that anthocyanin biosynthesis is closely associated with developmental stages of the fruit. In strawberry, flavonoid-3-O-glucosyltransferase activity is not essential for redirection from flavonol to anthocyanin formation during fruit ripening (Halbwirth et al. 2006). It has been shown that flavonoid enzyme activity peaks during fruit ripening at early and late developmental stages (Halbwirth et al. 2006). In grape, an increase in UFGT activity is concomitant with a rise in anthocyanin biosynthesis (Mori et al. 2005). This indicates that UFGT is required for anthocyanin biosynthesis in red coloration of many fruits. This close correlation between UFGT activity and anthocyanin content suggests a causal relation. Therefore, a key step for regulation of anthocyanin biosynthesis in
Malay apple was likely to be at the final step of the pathway controlled by UFGT rather than a step controlled by PAL.

The transcripts of SmPAL, SmCHS, SmCHI, SmF3H, $S m D F R$, and SmLDOX in both the wild-type and the mutant of Malay apple 'Saraek', were comparatively abundant throughout the fruit growth and ripening period. This suggests SmPAL, SmCHS, SmCHI, SmF3H, $S m D F R$, and $S m L D O X$ are not key genes regulating anthocyanin biosynthesis in Malay apple. It was reported that expression of FIPAL6 during fruit ripening correlates with the rapid accumulation of anthocyanin in strawberry (Pombo et al. 2011). In contrast, transcript abundances of $C H I$ and $D F R$ genes were high during the early stage of fruit development whereas there was no anthocyanin accumulation in strawberry (Li et al. 2001).

The transcription of SmUFGT increased markedly with red coloration during fruit growth and ripening. In the wild-type Malay apple 'Mamieo', the largest increase (more than 160-fold) was found in the transcription of the SmUFGT from stage 3 to stage 7 and a decline at the final stage (stage 8) whereas the largest increase (more than 80 -fold) from stage 3 to stage 8 was found in the wildtype Malay apple 'Saraek'. Those increases in SmUFGT expressions were correlated with an increase in fruit skin color, the total anthocyanin content, cyanidin-3-Oglucoside content, and UFGT activity. The expression pattern of the SmUFGT was similar to the expression of an UFGT in strawberry fruit (Almeida et al. 2007). The UFGT catalayzes anthocyanidin glycosylation at a final step of the anthocyanin biosynthetic pathway in grape fruit skin, which contains a high anthocyanin content. The role of UFGT in regulating anthocyanin accumulation in apple skin has been previously reported (Honda et al. 2002, Kondo et al. 2002, Zhang et al. 2013). Some studies reported a specific correlation between anthocyanin biosynthesis and UFGT expression (Kondo et al. 2002), other studies also reported the correlation of UFGT expression with other anthocyanin biosynthetic genes (Honda et al. 2002). Most of the anthocyanins in apple skin are galactosylated. It has been suggested that the UFGT gene isolated from apples encodes a galactosyl-transferase (Honda et al. 2002, Kondo et al. 2002). Walker et al. (2007) found that white grapes show no UFGT activity. They found that the white grapes have a detectable but

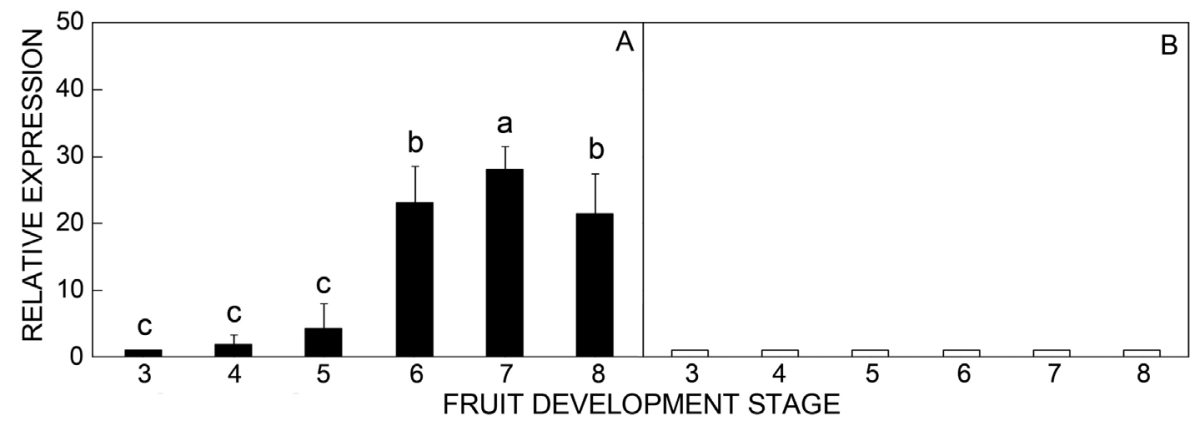

Fig. 7. Relative expression of the myeloblastosis transcription factor $(A, B)$ in Malay apple wild-type ( $\square)$ and mutant ( $\square$ ) fruits during their growth and ripening. Real-time PCR was used to analyze expression patterns. The column heights indicate relative mRNA abundances relative to stage 3, which was set to 1.0. All real time-PCR reactions were normalized using the actin gene. Means $\pm \mathrm{SEs}, n=3$. Different letters indicate significant differences according to the Duncan's new multiple range test $(P<0.05)$. 
very low amounts of UFGT transcripts. The sequence of the transcripts in the red and white grapes is the same showing that the mutation is not in the UFGT gene. It was established that a mutation in a MYB transcription factor is the cause of a low anthocyanin content. In contrast, we found no detectable UFGT transcription in the Malay apple mutant. This indicates that the mutation was either in the gene itself or in the transcription factor.

During red coloration, the transcription of the SmMyb increased markedly with onset of red coloration during fruit development. This expression pattern is similar to that of MdMYB10 in apple (Espley et al. 2007) and also similar to that of $V v M Y B P A 1$ in grape berry skins (Bogs et al. 2007). Expression patterns therefore suggest that $G m M Y B$ is a candidate to regulate anthocyanin biosynthesis of Malay apple fruits. The expression patterns of anthocyanin biosynthesis genes correlated with that of $G m M Y B 10$, which increases strongly with the onset of color development. In grape, $V v M Y B A 1$ and $V v M Y B A 2$ regulate specifically the expression of the UFGT gene (Kobayashi et al. 2002, Walker et al. 2007). In other species, regulation of anthocyanin biosynthesis has been shown to be at the level of transcription in pigmented organs other than fruits (Mol et al. 1998, Schwinn et al. 2006) and transcription indicates a final phenotype including color, and anthocyanin content and composition (Castellarin and Di Gaspero 2007).

In conclusion, the present work shows that cyanidin3-O-glucoside was the major anthocyanin in the red/ purple skin of the fruits of Malay apple. The cyanidin3-O-glucoside content markedly increased during wildtype fruit growth and maturation. This increase was correlated with color development, the activity of UFGT (the enzyme that apparently catalyses the last step of anthocyanin synthesis), and the expression of SmUFGT. The mutant, with a white fruit epidermis, lacked detectable anthocyanins, had no detectable UFGT activity, and no detectable expressions of SmUFGT and SmMyb. The $S m M Y B$ appears to play a role in the specific regulation of anthocyanin biosynthesis in Malay apple fruits. The SmUFGT may be a key gene in Malay apple pigmentation.

\section{References}

Almeida, J.R.M., D'Amico, E., Preuss, A., Carbone, F., De Vos, C.H.R., Deiml, B., Mourgues, F., Perrotta, G., Fischer, T.C., Bovy, A.G., Martens, S., Rosati, C.: Characterization of major enzymes and genes involved in flavonoid and proanthocyanidin biosynthesis during fruit development in strawberry (Fragaria ananassa). - Arch. Biochem. Biophys. 465: 61-71, 2007.

Ashton, M.B.O., Marie, W., Tonyk, M., Rosheila, V., Wang, Y., Requejo-Jackman, C., Ramakutty, P., Woolf, A.B.: Pigments in avocado tissue and oil. - J. Agr. Food Chem. 54: 10151-10158, 2006.

Awad, M.A., De Jager, A.: Relationships between fruit nutrients and concentrations of flavonoids and chlorogenic acid in 'Elstar' apple skin. - Sci. Hort. 92: 265-276, 2002.

Bogs, J., Jaffe, F.W., Takos, A.M., Walker, A.R., Robinson, S.P.: The grapevine transcription factor VvMYBPA1 regulates proanthocyanidin synthesis during fruit development. - Plant
Physiol. 143: 1347-1361, 2007.

Bradford, M.M.: A rapid and sensitive method for the quantitation of microgram quantities of protein utilizing the principle of protein-dye binding. - Anal. Biochem. 72: 248-254, 1976.

Broun, P.: Transcriptional control of flavonoid biosynthesis: a complex network of conserved regulators involved in multiple aspects of differentiation in Arabidopsis. - Curr. Opin. Plant Biol. 8: 272-279, 2005.

Castellarin, S., Di Gaspero, G.: Transcriptional control of anthocyanin biosynthetic genes in extreme phenotypes for berry pigmentation of naturally occurring grapevines. - BMC Plant Biol. 7: 46, 2007.

Cox, K.A. McGhie, T.K., White, A., Woolf, A.B.: Skin colour and pigment changes during ripening of 'Hass' avocado fruit. - Postharvest Biol. Technol. 31: 287-294, 2004.

Cronje, R.B.: Effect of fruit development, maturity and harvesting of litchi (Litchi chinensis Sonn.) on postharvest fruit quality. Stewart Postharvest Rev. 4: 1-10, 2008.

Espley, R.V., Hellens, R.P., Putterill, J., Stevenson, D.E., KuttyAmma, S., Allan, A.C.: Red colouration in apple fruit is due to the activity of the MYB transcription factor, MdMYB10. - Plant J. 49: 414-427, 2007.

Fu, C., Loo, A.E.K., Chia, F.P.P., Huang, D.: Oligomeric proanthocyanidins from mangosteen pericarps. - J. Agr. Food Chem. 55: 7689-7694, 2007.

Halbwirth, H., Puhl, I., Haas, U., Jezik, K., Treutter, D., Stich, K.: Two-phase flavonoid formation in developing strawberry $($ Fragaria $\times$ ananassa $)$ fruit. - J. Agr. Food Chem. 54: 1479$1485,2006$.

Honda, C., Kotoda, N., Wada, M., Kondo, S., Kobayashi, S., Soejima, J., Zhang, Z., Tsuda, T., Moriguchi, T.: Anthocyanin biosynthetic genes are coordinately expressed during red coloration in apple skin. - Plant Physiol. Biochem. 40: 955962, 2002.

Ji, X., Avula, B., Khan, I.A.: Quantitative and qualitative determination of six xanthones in Garcinia mangostana L. by LC-PDA and LC-ESI-MS. - J. Pharm. Biomed. Anal. 43: 1270-1276, 2007.

Ju, Z.G., Liou, C.L., Yuan, Y.B.: Activities of chalcone synthase and UDP Gal: flavonoid-3- $O$-glycosyltransferase in relation to anthocyanin synthesis in apple. - Sci. Hort. 63: 175-185, 1995a.

Ju, Z.G., Yuan,Y.B., Liou, C.L., Xin, S.H.: Relationships among phenylalanine ammonia-lyase activity, simple phenol concentrations and anthocyanin accumulation in apple. - Sci. Hort. 61: 215-226, 1995 b.

Kalt, W., Law, C., Ryan, D., McDonald, J.E., Donner, H.: Oxygen radical absorbing capacity, anthocyanin and phenolic content of high bush blueberries (Vaccinium corymbosum L.), during ripening and storage. - J. amer. Soc. hort. Sci. 128: 917-923, 2003.

Kobayashi, S., Ishimaru, M., Ding, C.K., Yakushiji, H., Goto, N., Comparison of UDP-glucose: flavonoid 3-O-glucosyltransferase (UFGT) gene sequences between white grapes (Vitis vinifera) and their sports with red skin. Plant Sci. 160: 543-550, 2001.

Kobayashi, S., Ishimaru, M., Hiraoka, K., Honda, C.: Mybrelated genes of the Kyoho grape (Vitis labruscana) regulate anthocyanin biosynthesis. - Planta 215: 924-933, 2002.

Kobayashi, S., Goto, N., Yakushiji, H., Retrotransposon-induced mutation in grape skin color. - Science 304: 982, 2004.

Koes, R., Verweij, W., Quattrocchio, F.: Flavonoids: a colorful model for the regulation and evolution of biochemical pathways. - Trends Plant Sci. 10: 236-242, 2005.

Kondo, S., Hiraoka, K., Kobayashi, S., Honda, C., Terahara, N.: Changes in the expression of anthocyanin biosynthetic genes 
during apple development. - J. amer. Soc. hort. Sci. 127: 971976, 2002.

Kotepong, P., Ketsa, S., Van Doorn, W.G.: A white mutant of Malay apple fruit (Syzygium malaccense) lacks transcript expression and activity for the last enzyme of anthocyanin synthesis, and the normal expression of a MYB transcription factor. - Funct. Plant Biol. 38: 75-86, 2011.

Lee, J.H., Talcott, S.T.: Fruit maturity and juice extraction influences ellagic acid derivatives and other antioxidant polyphenolics in muscadine grapes. - J. Agr. Food Chem. 52: 361-366, 2004.

Li, Y.J., Sakiyama, R., Maruyama, H., Kawabata, S.: Regulation of anthocyanin biosynthesis during fruit development in 'Nyoho' strawberry. - J. jap. Soc. hort. Sci. 70: 28-32, 2001.

Lister, C.E., Lancaster, J.E., Walker, J.R.L.: Developmental changes in enzymes of flavonoid biosynthesis in the skins of red and green apple cultivars. - J. Sci. Food Agr. 71: 313-330, $1996 \mathrm{a}$.

Lister, C.E., Lancaster, J.E., Walker, J.R.L.: Phenylalanine ammonia-lyase (PAL) activity and its relationship to anthocyanin and flavonoid levels in New Zealand-grown apple cultivars. - J. amer. Soc. hort. Sci. 121: 281-285, 1996b.

Liu, Y., Che, F., Wang, L., Meng, R., Zhang, X., Zhao, Z.: Fruit coloration and anthocyanin biosynthesis after bag removal in non-red and red apples (Malus $\times$ domestica Borkh.). Molecules 18: 1549-1563, 2013.

López-Gómez, R., Gómez-Lim, M.A.: A method for extracting intact RNA from fruits rich in polysaccharides using ripe mango mesocarp. - HortScience 27: 440-442, 1992.

Matus, J.T., Aquea, F., Arce-Johnson, P.: Analysis of the grape MYB R2R3 subfamily reveals expanded wine quality-related clades and conserved gene structure organization across Vitis and Arabidopsis genomes. - BMC Plant Biol. 8: 83, 2008.

Mol, J., Grotewold, E., Koes, R.: How genes paint flowers and seeds. - Trends Plant Sci. 3: 212-217, 1998.

Mori, K., Sugaya, S., Gemma, H.: Decreased anthocyanin biosynthesis in grape berries grown under elevated night temperature condition. - Sci. Hort. 105: 319-330, 2005.

Mori, K., Sugaya, S., Gemma, H.: Regulatory mechanism of anthocyanin biosynthesis in 'Kyoho' grape berries grown under different temperature conditions. - Environ. Control Biol. 42: 21-30, 2004.

Palapol,Y., Ketsa, S., Lin-Wang, K., Ferguson, I.B., Allan, A.C.: A MYB transcription factor regulates anthocyanin biosynthesis in mangosteen (Garcinia mangostana L.) fruit during ripening. - Planta 229: 1323-1334, 2009a.

Palapol, Y., Ketsa, S., Stevenson, D., Cooney, J.M., Allan, A.C., Ferguson, I.B.: Colour development and quality of mangosteen (Garcinia mangostana L.) fruit during ripening and after harvest. - Postharvest Biol. Technol. 51: 349-353, 2009b.

Piccaglia, R., Marotti, M., Baldoni, G.: Factors influencing anthocyanin content in red cabbage (Brassica oleracea var. capitata L f rubra (L) Thell). - J. Sci. Food Agr. 82: 15041509, 2002.

Pombo, M.A, Martínez G.A., Civello, P.M.: Cloning of FaPAL6 gene from strawberry fruit and characterization of its expression and enzymatic activity in two cultivars with different anthocyanin accumulation. - Plant Sci. 18: 111-118, 2011.

Schwinn, K., Venail, J., Shang, Y., Mackay, S., Alm, V., Butelli, E., Oyama, R., Bailey, P., Davies, K., Martin, C.: A small family of MYB-regulatory genes controls floral pigmentation intensity and patterning in the genus Antirrhinum. - Plant Cell 18: 831-851, 2006.

Segade, S.R., Vázquez, E.S., Losada, E.D.: Influence of ripeness grade on accumulation and extractability of grape skin anthocyanin in different cultivars. - J. Food Composition Anal. 21: 599-607, 2008.

Selahle, K.M., Sivakumar, D., Jifon, J., Soundy, P.: Postharvest responses of red and yellow sweet peppers grown under photo-selective nets. - Food Chem. 173: 951-956, 2015.

Sivankalyani, V., Feygenberg, O., Diskin, S., Wright, B., Alkan, N.: Increased anthocyanin and flavonoids in mango fruit peel are associated with cold and pathogen resistance. - Postharvest Biol. Technol. 111: 132-139, 2016.

Stevenson, D.E., Wibisono, R., Jensen, D.J., Stanley, R.A., Cooney, J.M.: Direct acylation of flavonoid glycosides with phenolic acids catalysed by Candida antarctica lipase B (Novozym 435 ${ }^{\circledR}$ ). - Enzyme Microbiol. Technol. 39: 12361241, 2006

Stracke, R., Werber, M., Weisshaar, B.: The R2R3-MYB gene family in Arabidopsis thaliana. - Curr. Opin. Plant Biol. 4: 447-456, 2001.

Takos, A.M., Jave, F.W., Jacob, S.R., Bogs, J., Robinson, S.P., Walke, A.R.: Light-induced expression of a $M Y B$ gene regulates anthocyanin biosynthesis in red apples. - Plant Physiol. 142: 1216-1232, 2006.

Vogt, T., Phenylpropanoid biosynthesis. - Mol. Plant 3: 2-20, 2010.

Walker, A.R., Lee, E., Bogs, J., McDavid, D.A.J., Thomas, M.R., Robinson, S.P.: White grapes arose through the mutation of two similar and adjacent regulatory genes. - Plant J. 49: 772$785,2007$.

Wang, H., Arakawa, O., Motomura, Y.: Influence of maturity and bagging on the relationship between anthocyanin accumulation and phenylalanine ammonia-lyase (PAL) activity in 'Jonathan' apples. - Postharvest Biol. Technol. 19: 123-128, 2000.

Whale, S.K., Singh, Z.: Endogenous ethylene and colour development in the skin of 'Pink lady' apple. - J. amer. Soc. hort. Sci. 132: 20-28, 2007.

Winkel-Shirley, B.: Biosynthesis of flavonoids and effects of stress. - Curr. Opin. Plant Biol. 5: 218-223, 2002.

Xie, R., Zheng, L., He, S., Zheng, Y., Yi, S., Deng, L.: Anthocyanin biosynthesis in fruit tree crops: genes and their regulation. Afr. J. Biotechnol. 10: 19890-19897, 2011.

Xu, F., Cao, S., Shi, L., Chen, W., Su, X., Yang, Z.: Blue light irradiation affects anthocyanin content and enzyme activities involved in postharvest strawberry fruit. - J. Agr. Food Chem. 62: 4778-4783, 2014.

Zhang, X.J., Wang, L.X., Liu, Y.L., Chen, X.X., Yang, Y.Z., Zhao, Z.Y.: Differential gene expression analysis of 'Granny Smith' apple (Malus domestica Borkh.) during fruit skin coloration. - South Afr. J. Bot. 88: 125-131, 2013. 